\title{
CLINICAL INDICATORS OF TREATMENT-RESISTANT PSYCHOSIS
}

Sophie E. Legge ${ }^{1}$, Charlotte A. Dennison ${ }^{1}$, Antonio F. Pardiñas ${ }^{1}$, Elliott Rees ${ }^{1}$, Amy J. Lynham ${ }^{1}$, Lucinda Hopkins $^{1}$, Lesley Bates ${ }^{1}$, George Kirov ${ }^{1}$, Michael J. Owen ${ }^{1}$, Michael C. O'Donovan ${ }^{1}$ \& James T.R. Walters ${ }^{1}$

Corresponding author

Professor James T.R. Walters

Deputy Director, National Centre for Mental Health

Division of Psychological Medicine and Clinical Neurosciences, School of Medicine, Cardiff University, Hadyn Ellis Building, Maindy Road, Cathays, Cardiff, CF24 4HO

Email:WaltersJT@cardiff.ac.uk

\section{Affiliations}

${ }^{1}$ MRC Centre for Neuropsychiatric Genetics and Genomics, Division of Psychological Medicine and Clinical Neurosciences, School of Medicine, Cardiff University, Cardiff, UK 


\begin{abstract}
Background

Around $30 \%$ of individuals with schizophrenia remain symptomatic and markedly impaired despite standard antipsychotic treatment and are considered to be treatment-resistant. Clinicians are currently unable to predict which patients are at higher risk of treatmentresistance.
\end{abstract}

Aims

To determine whether genetic liability for schizophrenia and/or clinical characteristics measurable at illness onset can prospectively indicate a higher risk of treatment-resistant psychosis (TRP).

\title{
Method
}

In 1070 individuals with schizophrenia or related psychotic disorders, schizophrenia polygenic risk scores (PRS) and large copy number variation (CNVs) were assessed for enrichment in TRP. Regression and machine-learning approaches were used to investigate the association of phenotypes related to demographics, family history, premorbid factors, and illness onset with TRP.

\section{Results}

A younger age of onset of psychosis (OR=0.94, $\left.p=7.79 \times 10^{-13}\right)$ and poor premorbid social adjustment $\left(\mathrm{OR}=1.64, p=2.41 \times 10^{-4}\right)$ increased the risk of TRP in univariate regression analyses. These factors remained associated in multivariate regression analyses, which also found lower premorbid IQ (OR=0.98, $\left.p=7.76 \times 10^{-3}\right)$, a younger father's age at birth (OR=0.97, $\left.p=0.015\right)$, and cannabis use $(\mathrm{OR}=1.60, p=0.025)$ to increase the risk of TRP. Machine-learning approaches 
found age at onset of psychosis to be the most important predictor and also identified premorbid IQ and poor premorbid social adjustment as predictors of TRP mirroring the findings from the regression analyses. Genetic liability for schizophrenia indicated by PRS and CNVs were not associated with TRP.

\section{Conclusions}

Patients with an earlier age of onset of psychosis and poor premorbid functioning are more likely to be treatment-resistant. The genetic architecture of susceptibility to schizophrenia may be distinct from that of treatment outcomes.

\section{Introduction}

Around $30 \%$ of individuals with schizophrenia will remain symptomatic and significantly impaired despite standard antipsychotic treatment and are considered to have treatmentresistant schizophrenia ${ }^{1}$. Treatment-resistance is usually defined as a failure to respond to two antipsychotic trials of sufficient dose and duration and is one of the most disabling forms of illness, and thus presents a major clinical challenge ${ }^{2}$. It is as yet unclear whether treatmentresistance is better conceptualised as a form of illness at the severe end of a spectrum, or as a more biologically homogeneous subgroup of those with schizophrenia, although recent evidence has supported the latter hypothesis ${ }^{3}$. Clozapine is the only medication with proven effectiveness for patients with treatment-resistant schizophrenia ${ }^{4}$. Nonetheless, substantial delays in receiving clozapine treatment are commonplace ${ }^{5}$, and these delays are associated with poorer outcomes ${ }^{6}$. Ensuring the right patients have timely access to clozapine is an important therapeutic goal in the management of people with schizophrenia? 
Although previous studies have identified clinical indicators of poor outcome in general, there have been relatively few studies investigating risk factors for treatment-resistance specifically. The research that has been undertaken indicates that an early age of onset of psychosis, male sex, a longer duration of untreated psychosis, and poor premorbid functioning may be associated with treatment-resistance ${ }^{8-10}$. Although one study reported an enrichment of schizophrenia PRS in clozapine-treated patients ${ }^{10}$, this has not been replicated in larger subsequent studies ${ }^{11,12}$. Other studies have reported an increased burden of genome-wide rare copy number duplications in treatment-resistant patients ${ }^{12}$, and an excess of rare disruptive variants in gene targets of antipsychotics ${ }^{13}$, although both of these studies are yet to be independently replicated. The identification of reliable factors could serve to alert clinicians and help predict those at greater risk of developing treatment-resistance when they first present with psychosis. This study aims to gain insights and identify factors measurable at illness onset that could be used predict treatment-resistant psychosis (TRP).

\section{Methods}

\section{Sample characteristics}

Study individuals were from the CardiffCOGS (COGnition in Schizophrenia, $n=1070$ ) sample, which has been previously described ${ }^{14,15}$ and additional details are provided in Supplementary Methods. CardiffCOGS is a sample of patients with clinically diagnosed schizophrenia or related psychotic disorders recruited from community, in-patient and voluntary sector mental health services in the UK. Study individuals completed a comprehensive clinical interview based on the Schedules for Clinical Assessment in Neuropsychiatry (SCAN) instrument, donated a blood sample for genetic analysis, and consented for access to their clinical case notes. The SCAN interview and clinical case notes were used to arrive at DSM-IV and ICD-10 lifetime diagnoses and to complete OPCRIT ratings. All study individuals had a diagnosis of schizophrenia or related 
psychotic disorder (detailed in Supplementary Table 1). We included individuals with related psychotic disorders in addition to those with schizophrenia given that the study focuses on prediction at first presentation and diagnosis can be uncertain at this time. Thus, we refer to all samples as either TRP or non-TRP (whether the diagnosis is schizophrenia or a related psychotic disorder) and we undertake sensitivity analyses for all results to examine whether effects are consistent when restricted to those with narrowly defined schizophrenia. The sample shows a degree of enrichment for TRP as a result of targeted recruitment from clozapine clinics $(52.4 \%$ compared to a TRP prevalence of $30 \%$ ). The study had multi-site NHS ethics approval granted by South East Wales Research Ethics Committee Panel (REC reference number: 07/WSE03/110) and written informed consent was obtained for all study participants.

\section{Outcome variable}

Study individuals were diagnosed with TRP if they had either been rated negatively for OPCRIT item 89 'psychotic symptoms respond to neuroleptics' or had received clozapine treatment. Individuals were diagnosed with non-TRP if they were rated positively for OPCRIT item 89 and had not received clozapine. OPCRIT item 89 was rated globally over the total period of illness based on interview and clinical notes data and scored positively if the illness appeared to respond to any type of antipsychotic or if relapse occurred when medication was stopped. Individuals were excluded from the analyses $(n=82)$ if (i) despite failure to respond to antipsychotics, they had not yet received two adequate antipsychotic trials at time of data collection or (ii) there was insufficient information to determine antipsychotic response.

\section{Demographic, premorbid and illness onset clinical factors}

To investigate characteristics that could at illness onset prospectively indicate a higher risk of TRP, we investigated variables related to demographics and family background, premorbid factors, and characteristics related to illness onset. These variables were derived from self- 
report at interview, clinical case notes and OPCRIT ratings. Full definitions of these variables are given in Supplementary Table 2. The demographic factors assessed included; (i) sex, (ii) urbanicity, (iii) family history of schizophrenia, (iv) family history of a psychotic disorder, affective disorder or suicide, (v) mother's age at birth, and (vi) father's age at birth. The premorbid factors investigated included; (i) birth complications, (ii) complications during their mother's pregnancy, (iii) developmental problems, (iv) childhood abuse, (v) total years spent in education, (vi) highest level of education, (vii) premorbid IQ, (viii) poor premorbid social adjustment, and (ix) poor premorbid work adjustment. Features related to the first illness presentation included; (i) age of onset of psychosis, (ii) duration of untreated psychosis, (iii) regular cannabis use in the year prior to illness onset, (iv) regular cigarette smoking in the year prior to illness onset, (v) a psychosocial stressor in the six months prior to onset of psychosis, and (vi) the mode of onset of psychosis.

\section{Lifetime characteristics}

In a secondary analysis, we investigated differences in post-onset symptom and outcome measures between individuals with TRP and non-TRP. These variables included demographics, lifetime clinical characteristics, clinical symptoms, and substance use (full details in Supplementary Table 3).

Genetic liability for schizophrenia

\section{Genotyping and quality control}

The CardiffCOGS sample was genotyped on either the Illumina HumanOmniExpressExome-8 or the Illumina HumanOmniExpress-12 array as previously described ${ }^{15}$. After standard $Q C$ procedures, imputation was performed using IMPUTE2 ${ }^{16}$ and the 1000 Genomes (phase 3 ) and UK10K reference panels ${ }^{17}$. Genetic analyses were restricted to those of European ancestry, 
assessed by principal component analysis, and related individuals with $\pi>0.2$ were identified and one member removed at random. Complete details of genotyping and quality control of the CardiffCOGS sample are provided in a prior publication ${ }^{15}$ and Supplementary Methods.

\section{Schizophrenia polygenic risk score (PRS)}

PRS were created based on the largest published schizophrenia GWAS meta-analysis ${ }^{15}$, excluding individuals from CardiffCOGS. Scores were calculated following the method described by Wray et $\mathrm{al}^{18}$ (Supplementary Methods). We selected nine $p$-value thresholds $\left(5 \times 10^{-8}, 1 \times 10^{-}\right.$ $\left.{ }^{6}, 1 \times 10^{-4}, 0.001,0.01,0.05,0.1,0.2,0.5\right)$ to compute PRS scores.

\section{Copy number variation (CNVs)}

The identification and quality control of CNVs in the CardiffCOGS sample has been previously described ${ }^{19}$ and detailed in Supplementary Methods. To compare the enrichment of rare, pathogenic CNVs in TRP with non-TRP, we analysed the presence of an intellectual disability associated (ID) $\mathrm{CNV}^{20}$, the presence of a CNV previously associated with schizophrenia ${ }^{19}$, and the presence of any chromosomal deletions and duplications spanning $500 \mathrm{~kb}$ or $1 \mathrm{Mb}$ in length.

\section{Analysis}

We conducted descriptive analyses to compare lifetime illness-related symptoms and outcome measures across TRP and non-TRP cases via univariate logistic regression. To assess the association of clinical predictive factors with TRP, we conducted univariate logistic regressions for each variable adjusting for age at interview and method of recruitment (defined as recruitment from secondary mental health care services such as clinician referral or clozapine clinic, or from other sources such as opportunistic or via third sector organisations). To control for multiple testing of 21 variables, we applied a Bonferroni correction threshold of $p \leq 2.38 \times 10^{-}$ 3. 
Multivariate prediction modelling of TRP consisted of two approaches (i) multivariate logistic regression including variables associated at $p<0.1$ from univariate analyses and covarying for age at interview and recruitment method, and (ii) conditional inference random forests model. Machine learning ensemble methods, such as the conditional inference random forests model, have been shown to have superior performance in detecting independent associations with health outcomes in comparison to logistic regression ${ }^{21}$. The conditional inference random forest model was implemented via the 'cforest' function in the $R$ 'party' package ${ }^{22}$, which is recommended for models that have variables of different types and that are correlated

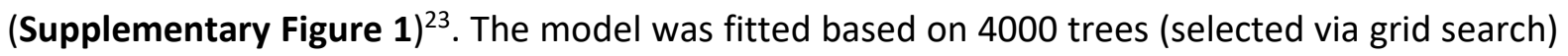
and an unbiased variable selection to control for different variable types. To derive an importance value for each variable, conditional permutation was used to control for any correlated variables. This permuted importance value represents the decrease in classification accuracy after randomly permuting the values of that variable over all trees. The accuracy of the conditional inference forest model was derived using the $\mathrm{R}$ 'caret' package ${ }^{24}$. The primary forest model could only be conducted in individuals with no missing data for the 21 variables analysed ( $n=337$ ), and so we repeated the analyses in the remaining sample (up to $n=733$ ) for the five variables with the highest importance from the primary analysis. In order to make a direct comparison between the logistic regression and conditional inference forest models, we conducted a multivariate logistic regression in the 337 individuals with complete data. Rates of missing data for each variable are detailed in Supplementary Table 2. The differences between individuals with and without missing data was assessed via logistic regression and detailed in Supplementary Table 4; there was no difference in sex, age at interview or method of recruitment but the participants with no missing data were less likely to have TRP $(O R=0.65$, $95 \% \mathrm{Cl}=0.50-0.84, p=0.001)$. 
To test the relationship between common variant genetic liability for schizophrenia on TRP, we regressed a model for each polygenic risk score created from various training $p$-value thresholds against a base model including the first five principal components and any additional principal components from the first 20 that were associated $(p<0.05)$ with TRP. To assess the proportion of variance explained we computed $R^{2}$ on the liability scale ${ }^{25}$, based on a TRP lifetime prevalence of $30 \%$ in schizophrenia, to account for ascertainment bias. The association of CNVs with TRP was tested via Firth's logistic regression ${ }^{26}$.

As this sample includes those with schizophrenia and related psychotic disorders, all analyses were replicated restricting the sample to those with a schizophrenia or schizoaffective disorder, depressed type diagnosis.

\section{Results}

A total 561 (52.4\%) of the individuals included in the study were diagnosed with TRP and 509 (47.6\%) with non-TRP. Due to our sampling methodology for this genetic study the vast majority of the participants (96.9\%) were of White European ethnicity, and a total of 662 (61.9\%) were male. Study individuals with TRP had a younger age at interview compared to those with non$\operatorname{TRP}\left(\mathrm{OR}=0.98,95 \% \mathrm{Cl}=0.97-0.99, p=6.48 \times 10^{-4}\right)$ and were more likely to have been recruited from secondary mental care services $\left(\mathrm{OR}=2.32,95 \% \mathrm{Cl}=1.76-3.04, p=1.52 \times 10^{-9}\right)$. As potential confounders of no experimental interest both of these factors were included as covariates in subsequent regression analyses.

\section{Lifetime characteristics}

In a descriptive comparison of post-onset symptom and outcome measures, we found that individuals with TRP were more severely impaired than those with non-TRP across a range of 
measures (Supplementary Table 5). Individuals with TRP were significantly more likely to have a continuous course of disorder, poorer cognitive functioning at the time of the interview, a higher number of psychiatric inpatient admissions, a lower GAS score, to have deteriorated from their premorbid level of functioning, to have been detained under the Mental Health Act, and to have a schizophrenia diagnosis. Furthermore, study individuals with TRP had more severe lifetime positive and negative symptoms. The strengths of the associations were equivalent in analyses restricted to individuals with schizophrenia or schizoaffective depression (Supplementary Table 6).

Demographic, premorbid and illness onset clinical factors

\section{Univariate logistic regression}

The association of demographic, premorbid and illness onset clinical factors with TRP are listed in Table 1. Univariate analyses in the total sample (up to $n=1070$ ) found significant associations with TRP for an earlier age of onset of psychosis ( $O R=0.94,95 \% \mathrm{Cl}=0.92-0.96, p=7.79 \times 10^{-13}$ ) and poor premorbid social adjustment $\left(\mathrm{OR}=1.64,95 \% \mathrm{Cl}=1.26-2.13, p=2.41 \times 10^{-4}\right)$. Lower premorbid IQ, poor premorbid work adjustment, and cannabis use in the year prior to illness onset were associated with TRP at $p<0.05$ but did not survive correction for multiple testing.

\section{Multivariate logistic regression}

Clinical factors associated at $p<0.1$ with TRP from the univariate analysis were included in a multivariate logistic regression (Table 1, $n=621$ ). We found that an earlier age of onset of psychosis $\left(\mathrm{OR}=0.95,95 \% \mathrm{Cl}=0.92-0.97, p=1.60^{-5}\right)$, poor premorbid social adjustment $(\mathrm{OR}=$ $\left.1.88,95 \% \mathrm{Cl}=1.27-2.78, p=1.49 \times 10^{-3}\right)$, lower premorbid IQ $(\mathrm{OR}=0.98,95 \% \mathrm{Cl}=0.96-0.99, p=$ $\left.7.76 \times 10^{-3}\right)$, younger father's age at birth $(\mathrm{OR}=0.97,95 \% \mathrm{Cl}=0.95-0.99, p=0.015)$, and cannabis use in the year prior to onset of psychosis $(\mathrm{OR}=1.60,95 \% \mathrm{Cl}=1.06-2.41, p=0.025)$ predicted 
TRP. The multivariate model explained $16.3 \%$ of the variance (Nagelkerke $\mathrm{R}^{2}$ of multivariate model minus that explained by covariates alone) of TRP. These associations remained consistent in regression analyses restricting the sample to individuals with schizophrenia and schizoaffective depression (Supplementary Table 7).

\section{Conditional inference forest model}

A conditional inference forest model was fitted to predict TRP based on 4000 trees using all 21 clinical factors previously described for 337 individuals with no missing data. The accuracy of the predictive model in this training dataset was 0.59 . Using conditional permutation for importance factors (which represents the decrease in classification accuracy if the values of that variable are randomly permuted), we found that a younger age of onset of psychosis was the most important factor in the prediction of TRP, followed by poor premorbid social adjustment, family history of schizophrenia, lower premorbid IQ and poor premorbid work adjustment (Figure 1). These findings were consistent in a model restricted to individuals with a diagnosis of schizophrenia (Supplementary Figure 2). The importance of these top five variables was replicated in 428 individuals that were excluded from the primary forest model analysis on the basis of having one or more of the 21 clinical factors missing. Age of onset of psychosis was again the most important factor for TRP prediction, followed by premorbid IQ and poor premorbid social adjustment. A logistic regression model for the 337 individuals included in the primary model is provided in Supplementary Table 8 for a direct comparison between the methods, although the findings from this restricted set are consistent with the primary regression analysis in any case.

[ Figure 1 approximately here ] 
Genetic liability for schizophrenia

\section{Schizophrenia polygenic risk score (PRS)}

Although individuals with TRP had a higher schizophrenia PRS on average across the p-value thresholds from the discovery cohort, this difference was only associated at a single threshold of $P<0.001$ (Table 2; $\left.R^{2}=0.011, O R=1.20,95 \% C l=1.03-1.39, p=0.016\right)$. These findings were consistent in analyses restricted to those with schizophrenia or schizoaffective depression (Supplementary Table 9). This study had $80 \%$ power to detect an association at $p<0.05$, if the correlation between genetic effects on schizophrenia and TRP was $49 \%{ }^{27}$.

\section{Copy number variation (CNVs)}

We found that there was no difference in the burden of rare, pathogenic CNVs previously associated with schizophrenia or intellectual disability in individuals with TRP compared to those with non-TRP (Table 2). Furthermore, we found no enrichment of $500 \mathrm{~kb}$ or $1 \mathrm{Mb}$ deletions or duplications in individuals with TRP. These findings were consistent in analyses restricted to those with schizophrenia or schizoaffective depression (Supplementary Table 10). Our sample size had $80 \%$ power to detect an OR $>1.7$ for a burden of CNVs with a frequency of $2.5 \%{ }^{28}$ at a significance level of $p<0.05^{29}$.

As genetic liability for schizophrenia (CNVs or PRS) was not associated with TRP, they were not combined in multivariate analyses with clinical factors.

Post hoc analyses related to age of onset of psychosis

Given the significant association of age of onset of psychosis with TRP, we conducted additional exploratory analyses. The association between TRP and age of onset of psychosis was consistent for males $\left(\mathrm{OR}=0.95,95 \% \mathrm{Cl}=0.93-0.97, p=3 \times 10^{-6}\right)$ and females $(\mathrm{OR}=0.93,95 \% \mathrm{Cl}=0.90-0.95$, $\left.p=7.9 \times 10^{-8}\right)$. To investigate whether clozapine-prescribing practice could be influencing these 
results, we restricted the analysis to TRP patients that had not received clozapine treatment ( $n=87)$ and found the association between age of onset of psychosis and TRP remained (OR = $\left.0.95,95 \% \mathrm{Cl}=0.92-0.98, p=5.88 \times 10^{-4}\right)$.

Age of onset of psychosis alone explained $7.3 \%$ of the variance (Nagelkerke $\mathrm{R}^{2}$ ) of TRP, and had an area under the curve of 0.65 . Figure 2 displays the relationship between age of onset and the proportion with TRP in our sample (data used given in Supplementary Table 11). Assuming a TRP prevalence of $30 \%$, we found that the positive predictive value for non-TRP was 0.51 for those with an age of onset less than 16 , and this increased to $0.60,0.66,0.73,0.79$, and 0.92 for ages of onset between 16-20, 21-25, 26-30, 31-40 and 41 years and over respectively (Supplementary Table 12).

We investigated whether the relationship between age of onset of psychosis and TRP could be explained by genetic liability to schizophrenia indicated by PRS and found that schizophrenia PRS was associated with the age of onset of psychosis (Supplementary Tables 13-14; at SNP threshold $p<0.01: \mathrm{R}^{2}=0.006$, Beta $\left.=-0.86,95 \% \mathrm{Cl}=-1.62--0.10, p=0.027\right)$. The association between age of onset of psychosis and TRP $\left(\mathrm{OR}=0.94,95 \% \mathrm{Cl}=0.92-0.96, p=4.60 \times 10^{-11}\right)$ was not attenuated when conditioning on schizophrenia PRS (OR $=0.94,95 \% \mathrm{Cl}=0.92-0.96, p=$ $\left.6.38 \times 10^{-11}\right)$.

[ Figure 2 approximately here ]

\section{Discussion}

In this study we used regression and machine-learning analyses to determine whether genetic liability for schizophrenia and/or clinical characteristics measurable at illness onset can prospectively indicate a higher risk of TRP. We found age of onset of psychosis to be a significant and important clinical indicator in TRP. In addition, we found evidence across models that poor premorbid social functioning and lower premorbid IQ increased the risk of TRP. Genetic liability 
for schizophrenia indicated by polygenic risk scores and rare, pathogenic CNVs were not associated with TRP and did not explain the relationship with age of onset of psychosis.

\section{Main findings}

Age of onset of psychosis, defined as the age at which treatment was first sought or when symptoms first caused significant impairment (if earlier), was the most important indicator of TRP in this study; it was significant in univariate and multivariate regression analyses $(O R=0.95$, $95 \% \mathrm{Cl}=0.92-0.97)$ and had the highest importance value from conditional inference forest model. These findings were consistent in analyses restricting the sample to individuals with a schizophrenia diagnosis and were not influenced by clozapine-prescribing practice. An early age of onset of psychosis has been previously identified in several pervious studies as a predictor of $\operatorname{TRP}^{8,9,12}$. This study provides evidence that the increased risk for TRP is not restricted to those with a very early onset as suggested by previous studies ${ }^{30,31}$ but rather the risk of TRP continued to reduce throughout adulthood; the positive predictive value for treatment response to standard antipsychotics increased from 0.51 for individuals with an age of onset below 16 to 0.92 for those with an age of onset over 41 years of age. Given the continued reduction in the risk of TRP throughout adulthood, it seems unlikely that the relationship is explained by differences in provision of health services or related factors across different age groups. Nonetheless, these findings do suggest the importance of engagement and proactive management of patients with an early age of onset of psychosis.

In this study we also found evidence for the role of premorbid factors in TRP. Poor premorbid social functioning, defined as a difficulty entering or maintaining social relationships, isolation or social withdrawal prior to the onset of psychotic symptoms, was significantly associated with TRP in univariate and multiple regression analyses $(\mathrm{OR}=1.88,95 \% \mathrm{Cl}=1.27-2.78)$ and rated as important in the conditional inference forest model. This finding is consistent with previous 
studies, which have identified premorbid social functioning as predictive of poor outcomes in schizophrenia and also TRP ${ }^{32}$. Furthermore, lower premorbid IQ (estimated from the National Adult Reading Test) was associated with an increased risk of TRP in multivariate regression analysis $(\mathrm{OR}=0.98,95 \% \mathrm{Cl}=0.96-0.99)$ and was identified by the conditional inference forest model as predictive. These premorbid factors have been implicated in other studies of poor outcomes in schizophrenia ${ }^{10,33}$, and warrant further investigation for their role in treatmentresistance. The association of premorbid factors with TRP suggests that the neurodevelopmental hypothesis of schizophrenia may have relevance to treatment outcomes and warrants further investigation. The multivariate logistic regression also found some evidence for the role of father's age at birth, and cannabis use in the year prior to onset of psychosis in TRP. However, these factors were not identified in the forest model and thus replication is required to investigate their role in TRP.

In this study we did not find evidence of an association between TRP and several established risk factors for schizophrenia such as male sex, childhood abuse, duration of untreated psychosis, family history of psychosis, or urbanicity. A lack of association with these variables has also been reported in other studies of $\mathrm{TRP}^{8,12}$, and provides support against a psychosis spectrum theory, under which you would expect to find increased rates of risk factors for schizophrenia in TRP. However, in the case of early adversity, a previous study reported a cumulative effect of lifetime adversity in $\mathrm{TRP}^{34}$, suggesting that although we did not find an independent association with childhood abuse, it is possible it could be contributing to TRP in a cumulative manner.

We found no evidence for the association of genetic liability for schizophrenia indicated by PRS or rare, pathogenic CNVs with TRP. These findings are consistent with other studies investigating the association of schizophrenia PRS with TRP ${ }^{10-12}$, suggesting that genetic liability to TRP is not strongly influenced by liability to schizophrenia, beyond of course the requirement 
to have schizophrenia. However, larger samples are required to provide definitive answers in this regard. Within the power limitations of the study design, our findings add support to other evidence $^{3}$ suggesting that treatment-resistance may not be best conceptualised as a form of illness at the severe end of a psychosis spectrum, in which case you would expect a higher genetic loading of generic schizophrenia-associated SNPs in TRP patients. The use of PRS in the personalised prediction of TRP may be better informed by more specific training sets, for example from genetic studies investigating TRP directly, as opposed to PRS derived from those with a broad schizophrenia diagnosis.

Schizophrenia PRS was weakly associated with age of onset of psychosis in this study. However, the relationship between TRP and age of onset of psychosis in this study was not confounded or explained by differences in genetic liability to schizophrenia, suggesting that genetic factors influencing the age of onset of psychosis are distinct from those that increase liability for the disorder and may have particular relevance to treatment-resistance.

\section{Lifetime characteristics}

In a descriptive comparison of post-onset symptom and outcome measures, we found that individuals with TRP were more severely impaired than those with non-TRP; they were significantly more likely to have a continuous course of disorder, poorer cognitive functioning at the time of the interview, a higher number of psychiatric inpatient admissions, a lower GAS score, were more likely to deteriorate from their premorbid level of functioning, more likely to have been detained under the Mental Health Act, and have more severe positive and negative symptoms. Many of these findings have been previously documented ${ }^{2}$, but few studies have looked at so many variables in a single cohort of this size. These findings reinforce the importance of efforts to identify early indicators of TRP and thus improve the ability of clinicians to identify and appropriately treat those with an increased risk of TRP. 


\section{Strengths and limitations}

A strength of the study is the use of a single large schizophrenia and related psychotic disorders sample with detailed clinical phenotypes derived from both interview and clinical case notes. Consequently, the outcome variable of TRP is of a high quality, including confirmed treatmentresponders as controls, which is not the case for many studies in this field. We did not have objective evidence (such as medication serum levels) to confirm treatment resistance but our TRP definition takes non-adherence into account given it relies upon either clinician diagnosis of treatment resistance to prescribe clozapine or was based on ratings from interview and clinical note review taking into account reported non-adherence. A further strength is the use of both regression and machine learning approaches. Machine learning models are increasingly being applied in prediction models for disease and to inform the personalised prevention of disease $\mathrm{e}^{35}$.

The primary limitation of this study is the use of retrospective reports for the premorbid and illness onset variables although the use of contemporaneous clinical records will have increased the reliability of the key clinical variables such as age of onset of psychosis. The study sample is enriched for individuals with TRP ( $52 \%$ vs. estimated $30 \%$ prevalence) as a result of targeted recruitment from clozapine clinics. Recruitment strategy was controlled for in regression analyses, which did not alter the results, and all predictive values were corrected for prevalence and thus we do not believe that this enrichment biases the results of the study. We found minor but consistent levels of missing data across clinical variables, which significantly reduced the sample size in multivariate analyses. However, we were able to replicate our findings in these excluded individuals for smaller subsets of associated variables. We were not able to incorporate some factors into the present study that may have impacted the likelihood of treatment-resistance such as type of individual antipsychotics prescribed and other treatments received. Lastly, like many genetic studies, our analyses primarily consisted of individuals of 
White European ethnicity and thus further studies are required to establish the generalisability of these findings to all TRP patients.

\section{Future research and clinical implications}

The results in this study indicate that genetic studies investigating TRP directly, rather than a broad schizophrenia diagnosis, will be needed to gain insights into the nature of treatmentresistance in schizophrenia and related psychotic disorders. Age of onset of psychosis, poor premorbid social functioning and premorbid IQ may serve as useful indicators, along with other factors, in a predictive algorithm for TRP. From a clinical perspective, the results indicate that patients with these characteristics are less likely to respond to standard antipsychotic treatment, and thus require additional monitoring, support, and perhaps clozapine treatment should be considered earlier. Measures of genetic liability to schizophrenia were not associated with TRP in this sample, indicating that the genetic architecture of susceptibility to schizophrenia may be distinct from that of treatment outcomes.

\section{Acknowledgements}

We thank the participants, clinicians, field team and MRC Centre for Neuropsychiatric Genetics and Genomics laboratory staff for their help with the CardiffCOGS study. We thank the staff at deCODE Genetics for sample genotyping.

\section{Declaration of Interest}

The authors declare no conflicts of interest. 


\section{Funding}

This project was supported by the following grants: Medical Research Council (MRC) Centre (MR/L010305/1), Program (G0800509), Project (MR/L011794/1) grants to Cardiff University, and The National Centre for Mental Health (NCMH), funded by the Welsh Government through Health and Care Research Wales.

\section{Author Contributions}

SL and JW conceived and designed the study. SL performed the analyses and drafted the manuscript. JW, MO and MOD supervised the project. $C D, A L, A P, E R, L H, L B$ and GK contributed to genetic and/or phenotypic data acquisition and preparation. All authors contributed to the revision and gave final approval of the manuscript.

\section{Author Details}

Sophie E. Legge, BSc, PhD, Research Associate, MRC Centre for Neuropsychiatric Genetics and Genomics, Division of Psychological Medicine and Clinical Neurosciences, Cardiff University, UK; Charlotte A. Dennison, BSc, PhD Student, MRC Centre for Neuropsychiatric Genetics and Genomics, Division of Psychological Medicine and Clinical Neurosciences, Cardiff University, UK; Antonio F. Pardiñas, BSc, PhD, Lecturer, MRC Centre for Neuropsychiatric Genetics and Genomics, Division of Psychological Medicine and Clinical Neurosciences, Cardiff University, UK; Elliott Rees, BSc, PhD, Research Associate, MRC Centre for Neuropsychiatric Genetics and Genomics, Division of Psychological Medicine and Clinical Neurosciences, Cardiff University, UK;

Amy J. Lynham, BSc, PhD, Research Associate, MRC Centre for Neuropsychiatric Genetics and Genomics, Division of Psychological Medicine and Clinical Neurosciences, Cardiff University, UK; 
Lucinda Hopkins, BSc, Sample and Governance Manager, MRC Centre for Neuropsychiatric Genetics and Genomics, Division of Psychological Medicine and Clinical Neurosciences, Cardiff University, UK;

Lesley Bates, BSc, Laboratory Manager, MRC Centre for Neuropsychiatric Genetics and Genomics, Division of Psychological Medicine and Clinical Neurosciences, Cardiff University, UK;

George Kirov, MRCPsych, PhD, Professor, MRC Centre for Neuropsychiatric Genetics and Genomics, Division of Psychological Medicine and Clinical Neurosciences, Cardiff University, UK;

Michael J. Owen, FRCPsych, PhD, Director, MRC Centre for Neuropsychiatric Genetics and Genomics, Division of Psychological Medicine and Clinical Neurosciences, Cardiff University, UK;

Michael C. O'Donovan, FRCPsych, PhD, Professor, MRC Centre for Neuropsychiatric Genetics and Genomics, Division of Psychological Medicine and Clinical Neurosciences, Cardiff University, UK;

James T.R. Walters, MRCPsych, PhD, Professor, MRC Centre for Neuropsychiatric Genetics and Genomics, Division of Psychological Medicine and Clinical Neurosciences, Cardiff University, UK

\section{Corresponding author}

Professor James T.R. Walters

Deputy Director, National Centre for Mental Health

Division of Psychological Medicine and Clinical Neurosciences, School of Medicine, Cardiff University, Hadyn Ellis Building, Maindy Road, Cathays, Cardiff, CF24 4HQ

Email: WaltersJT@cardiff.ac.uk 


\section{References}

1. Suzuki T, Remington G, Mulsant BH, et al. Defining treatment-resistant schizophrenia and response to antipsychotics: a review and recommendation. Psychiatry research 2012;197:1-6.

2. Kennedy JL, Altar CA, Taylor DL, Degtiar I, Hornberger JC. The social and economic burden of treatment-resistant schizophrenia: a systematic literature review. Int Clin Psychopharmacol 2014;29:63-76.

3. Gillespie AL, Samanaite R, Mill J, Egerton A, MacCabe JH. Is treatment-resistant schizophrenia categorically distinct from treatment-responsive schizophrenia? a systematic review. BMC Psychiatry 2017;17.

4. Leucht S, Corves C, Arbter D, Engel RR, Li C, Davis JM. Second-generation versus firstgeneration antipsychotic drugs for schizophrenia: a meta-analysis. The Lancet 2009;373:31-41.

5. Howes OD, Vergunst F, Gee S, McGuire P, Kapur S, Taylor D. Adherence to treatment guidelines in clinical practice: study of antipsychotic treatment prior to clozapine initiation. The British Journal of Psychiatry 2012;201:481-5.

6. Shah P, Iwata Y, Plitman E, et al. The impact of delay in clozapine initiation on treatment outcomes in patients with treatment-resistant schizophrenia: A systematic review. Psychiatry research 2018;268:114-22.

7. National Collaborating Centre for Mental Health. Psychosis and Schizophrenia in Adults: Treatment and Management. 2014.

8. Wimberley T, Stovring H, Sorensen HJ, Horsdal HT, MacCabe JH, Gasse C. Predictors of treatment resistance in patients with schizophrenia: a population-based cohort study. The lancet Psychiatry 2016;3:358-66.

9. Kim JS, Park CM, Choi JA, et al. The association between season of birth, age at onset, and clozapine use in schizophrenia. Acta Psychiatr Scand 2017;136:445-54.

10. Frank J, Lang $\mathrm{M}$, Witt $\mathrm{SH}$, et al. Identification of increased genetic risk scores for schizophrenia in treatment-resistant patients. Molecular psychiatry 2015;20:150-1.

11. Wimberley T, Gasse C, Meier SM, Agerbo E, MacCabe JH, Horsdal HT. Polygenic Risk Score for Schizophrenia and Treatment-Resistant Schizophrenia. Schizophr Bull 2017;43:1064-9.

12. Martin AK, Mowry B. Increased rare duplication burden genomewide in patients with treatment-resistant schizophrenia. Psychological medicine 2016;46:469-76.

13. Ruderfer DM, Charney AW, Readhead B, et al. Polygenic overlap between schizophrenia risk and antipsychotic response: a genomic medicine approach. The lancet Psychiatry 2016;3:350-7.

14. Lynham AJ, Hubbard L, Tansey $\mathrm{KE}$, et al. Examining cognition across the bipolar/schizophrenia diagnostic spectrum. Journal of Psychiatry and Neuroscience 2018;43:245-53.

15. Pardinas AF, Holmans $P$, Pocklington AJ, et al. Common schizophrenia alleles are enriched in mutation-intolerant genes and in regions under strong background selection. Nature genetics 2018.

16. Howie BN, Donnelly P, Marchini J. A flexible and accurate genotype imputation method for the next generation of genome-wide association studies. PLoS genetics 2009;5:e1000529.

17. Huang J, Howie B, McCarthy S, et al. Improved imputation of low-frequency and rare variants using the UK10K haplotype reference panel. Nat Commun 2015;6:8111.

18. Wray NR, Lee SH, Mehta D, Vinkhuyzen AA, Dudbridge F, Middeldorp CM. Research review: Polygenic methods and their application to psychiatric traits. Journal of Child Psychology and Psychiatry 2014;55:1068-87.

19. Rees E, Walters JT, Georgieva L, et al. Analysis of copy number variations at 15 schizophrenia-associated loci. The British Journal of Psychiatry 2014;204:108-14.

20. Coe BP, Witherspoon K, Rosenfeld JA, et al. Refining analyses of copy number variation identifies specific genes associated with developmental delay. Nature genetics 2014;46:1063-71.

21. Mansiaux Y, Carrat F. Detection of independent associations in a large epidemiologic dataset: a comparison of random forests, boosted regression trees, conventional and penalized 
logistic regression for identifying independent factors associated with H1N1pdm influenza infections. Bmc Med Res Methodol 2014;14.

22. Hothorn T, Kornik. K. \& Zeileis, A. party: A Laboratory for Recursive Part(y)itioning. 2006.

23. Strobl C, Hothorn T, Zeileis A. Party on! R J 2009;1:14-7.

24. Kuhn M. Building Predictive Models in R Using the caret Package. J Stat Softw 2008;28:1-26.

25. Lee SH, Goddard ME, Wray NR, Visscher PM. A better coefficient of determination for genetic profile analysis. Genetic Epidemiology 2012;36:214-24.

26. Wang $X$. Firth logistic regression for rare variant association tests. Frontiers in Genetics 2014;5:187.

27. Dudbridge F. Power and predictive accuracy of polygenic risk scores. PLoS genetics 2013;9:e1003348.

28. Rees E, Kendall K, Pardinas AF, et al. Analysis of Intellectual Disability Copy Number Variants for Association With Schizophrenia. JAMA psychiatry 2016;73:963-9.

29. Purcell S, Cherny SS, Sham PC. Genetic Power Calculator: design of linkage and association genetic mapping studies of complex traits. Bioinformatics 2003;19:149-50.

30. Lally J, Ajnakina O, Di Forti M, et al. Two distinct patterns of treatment resistance: clinical predictors of treatment resistance in first-episode schizophrenia spectrum psychoses. Psychological medicine 2016;46:3231-40.

31. Meltzer HY. Treatment-resistant schizophrenia--the role of clozapine. Curr Med Res Opin 1997;14:1-20.

32. Kanahara N, Yamanaka H, Suzuki T, Takase M, lyo M. First-episode psychosis in treatmentresistant schizophrenia: a cross-sectional study of a long-term follow-up cohort. BMC Psychiatry 2018;18:274.

33. Carbon $\mathrm{M}$, Correll CU. Clinical predictors of therapeutic response to antipsychotics in schizophrenia. Dialogues in clinical neuroscience 2014;16:505-24.

34. Hassan AN, De Luca V. The effect of lifetime adversities on resistance to antipsychotic treatment in schizophrenia patients. Schizophr Res 2015;161:496-500.

35. Koutsouleris N, Kambeitz-llankovic L, Ruhrmann S, et al. Prediction Models of Functional Outcomes for Individuals in the Clinical High-Risk State for Psychosis or With Recent-Onset Depression: A Multimodal, Multisite Machine Learning Analysis. JAMA psychiatry 2018;75:1156-72. 
Table 1: Demographic, premorbid and illness onset predictors of TRP

\begin{tabular}{|c|c|c|c|c|c|c|c|c|}
\hline & \multicolumn{2}{|l|}{ TRP } & \multicolumn{2}{|c|}{ Non-TRP } & \multicolumn{2}{|c|}{ Adjusted Univariate (up to $\mathrm{N}=1070$ ) } & \multicolumn{2}{|c|}{ Fully Adjusted Multivariate ( $\mathrm{N}=621$ ) } \\
\hline & $\mathrm{N}(\%) /$ mean (sd) & Total $\mathrm{N}$ & $\mathrm{N}(\%) /$ mean (sd) & Total $\mathrm{N}$ & OR $(95 \% \mathrm{Cl})$ & $P$ & OR $(95 \% \mathrm{Cl})$ & $P$ \\
\hline \multicolumn{9}{|l|}{ Demographics and family background } \\
\hline Male sex & $361(64.3 \%)$ & 561 & $300(59.1 \%)$ & 508 & $1.24(0.97-1.60)$ & 0.092 & $1.03(0.71-1.49)$ & 0.899 \\
\hline Urbanicity (city birth and upbringing) & $184(41.5 \%)$ & 443 & $175(38.8 \%)$ & 451 & $1.23(0.94-1.63)$ & 0.137 & & \\
\hline Family $\mathrm{Hx}$ of schizophrenia & $126(26.2 \%)$ & 481 & $92(20.8 \%)$ & 442 & $1.37(1.00-1.88)$ & 0.051 & $1.03(0.68-1.57)$ & 0.877 \\
\hline Family $\mathrm{Hx}$ of psychosis, affective or suicide & $255(54.6 \%)$ & 467 & $253(57.1 \%)$ & 443 & $0.86(0.67-1.13)$ & 0.276 & & \\
\hline Mother's age at birth & $26.71(\mathrm{sd}=6.3)$ & 490 & $27.16(\mathrm{sd}=6.1)$ & 475 & $0.99(0.97-1.01)$ & 0.466 & & \\
\hline Father's age at birth & $29.38(\mathrm{sd}=7.3)$ & 469 & $30.22(\mathrm{sd}=7.3)$ & 447 & $0.98(0.97-1.00)$ & 0.096 & $0.97(0.95-0.99)$ & 0.015 \\
\hline \multicolumn{9}{|l|}{ Premorbid factors } \\
\hline Birth complications & $113(23.8 \%)$ & 475 & $102(22.5 \%)$ & 453 & $1.09(0.79-1.49)$ & 0.604 & & \\
\hline Pregnancy complications & $48(10.4 \%)$ & 461 & $40(9.3 \%)$ & 432 & $1.18(0.75-1.86)$ & 0.477 & & \\
\hline Developmental problems & $100(20.4 \%)$ & 491 & 73 (15.9\%) & 460 & $1.32(0.94-1.86)$ & 0.109 & & \\
\hline Childhood abuse & $102(20.1 \%)$ & 508 & $93(19.5 \%)$ & 467 & $1.08(0.78-1.49)$ & 0.650 & & \\
\hline Years in education & $12.75(\mathrm{sd}=3.5)$ & 536 & $13.22(\mathrm{sd}=2.9)$ & 494 & $0.95(0.91-1.00)$ & 0.032 & $0.98(0.93-1.04)$ & 0.557 \\
\hline Highest level of education & $2.59(\mathrm{sd}=1.7)$ & 539 & $2.88(\mathrm{sd}=1.8)$ & 501 & $0.92(0.85-0.99)$ & 0.019 & $1.11(0.96-1.28)$ & 0.163 \\
\hline Premorbid IQ (NART) & $97.05(\mathrm{sd}=13.5)$ & 516 & $100.4(\mathrm{sd}=12.9)$ & 437 & $0.99(0.98-1.00)$ & $3.59 \times 10-3$ & $0.98(0.96-0.99)$ & $7.76 \times 10-3$ \\
\hline Poor premorbid social adjustment & $238(44.7 \%)$ & 532 & $163(33.7 \%)$ & 484 & $1.64(1.26-2.13)$ & $2.41 \times 10-4$ & $1.88(1.27-2.78)$ & $1.49 \times 10-3$ \\
\hline Poor premorbid work adjustment & $114(22.1 \%)$ & 515 & $71(14.8 \%)$ & 481 & $1.60(1.15-2.23)$ & $5.48 \times 10-3$ & $1.33(0.81-2.17)$ & 0.261 \\
\hline \multicolumn{9}{|l|}{ IIIness presentation } \\
\hline Definite psychosocial stressor within $6 \mathrm{~m}$ & $40(7.6 \%)$ & 524 & $54(11.2 \%)$ & 483 & $0.67(0.43-1.04)$ & 0.077 & $0.79(0.41-1.50)$ & 0.465 \\
\hline Age of onset of psychosis & $23.00(\mathrm{sd}=8.0)$ & 540 & $27.83(\mathrm{sd}=10.2)$ & 487 & $0.94(0.92-0.96)$ & $7.97 \times 10-13$ & $0.95(0.92-0.97)$ & $1.60 \times 10-5$ \\
\hline Duration of untreated psychosis (years) & $2.14(\mathrm{sd}=4.6)$ & 522 & $1.98(\mathrm{sd}=4.22)$ & 474 & $1.02(0.99-1.05)$ & 0.290 & & \\
\hline Cannabis use in year prior to onset & $186(36.1 \%)$ & 515 & $121(25.3 \%)$ & 479 & $1.53(1.14-2.06)$ & $5.13 \times 10-3$ & $1.60(1.06-2.41)$ & 0.025 \\
\hline Cigarette smoking prior to onset & $303(65.6 \%)$ & 462 & $285(62.0 \%)$ & 460 & $1.18(0.90-1.56)$ & 0.240 & & \\
\hline Insidious disease onset (1-6) & $3.64(\mathrm{sd}=1.4)$ & 433 & $3.49(\mathrm{sd}=1.4)$ & 428 & $1.05(0.95-1.15)$ & 0.377 & & \\
\hline
\end{tabular}


Table 1: Association of demographic, premorbid and illness onset clinical factors with treatment-resistant schizophrenia (TRP). Columns represent clinical variables, TRP, non-TRP (reference group), odds ratio (OR), 95\% confidence intervals $(\mathrm{Cl})$ and P-value from univariate logistic regression adjusted for age at interview and method of recruitment, and adjusted multivariate logistic regression. For binary variables, numbers $(\mathrm{N})$ and percentages (\%) are provided, and for continuous variables, mean and standard deviation (sd) are provided. P-values in bold were significant at $\mathrm{P}<0.05$. 
Table 2: Association of genetic liability for schizophrenia with TRP

\begin{tabular}{|c|c|c|c|c|c|c|}
\hline \multicolumn{7}{|c|}{ Schizophrenia polygenic risk score } \\
\hline Score P-value Threshold & \multicolumn{2}{|c|}{ OR $(95 \% \mathrm{Cl})$} & $\mathbf{R}^{2}$ & AUC & SE & P-value \\
\hline$P<1 \times 10-8$ & \multicolumn{2}{|c|}{$1.06(0.92-1.21)$} & 0.0011 & 0.515 & 0.0025 & 0.428 \\
\hline$P<1 \times 10-6$ & \multicolumn{2}{|c|}{$1.05(0.91-1.20)$} & 0.0007 & 0.513 & 0.0021 & 0.520 \\
\hline$P<1 \times 10-4$ & \multicolumn{2}{|c|}{$1.08(0.94-1.24)$} & 0.0021 & 0.521 & 0.0035 & 0.296 \\
\hline$P<1 \times 10-3$ & \multicolumn{2}{|c|}{$1.20(1.03-1.39)$} & 0.0110 & 0.549 & 0.0081 & 0.016 \\
\hline$P<0.01$ & \multicolumn{2}{|c|}{$1.11(0.94-1.30)$} & 0.0030 & 0.526 & 0.0043 & 0.212 \\
\hline$P<0.05$ & \multicolumn{2}{|c|}{$1.09(0.92-1.31)$} & 0.0020 & 0.521 & 0.0035 & 0.319 \\
\hline$P<0.1$ & \multicolumn{2}{|c|}{$1.07(0.89-1.28)$} & 0.0011 & 0.517 & 0.0026 & 0.469 \\
\hline$P<0.2$ & \multicolumn{2}{|c|}{$1.13(0.94-1.37)$} & 0.0032 & 0.526 & 0.0044 & 0.204 \\
\hline$P<0.5$ & \multicolumn{2}{|c|}{$1.08(0.89-1.31)$} & 0.0013 & 0.517 & 0.0028 & 0.441 \\
\hline \multicolumn{7}{|c|}{ Copy number variation } \\
\hline & & \multicolumn{2}{|c|}{$\begin{array}{l}\text { TRP N (\%) } \\
\text { Total = 429 }\end{array}$} & $\begin{array}{l}\text { Non-TRP N (\%) } \\
\text { Total = } 411\end{array}$ & OR (95\% Cl) & $\mathbf{P}$ \\
\hline \multicolumn{2}{|c|}{ Intellectual disability pathogenic CNV } & \multicolumn{2}{|c|}{$9(2.1 \%)$} & $11(2.7 \%)$ & $0.79(0.32-1.88)$ & 0.588 \\
\hline \multicolumn{2}{|c|}{ Schizophrenia pathogenic CNV } & \multicolumn{2}{|c|}{$7(1.6 \%)$} & $11(2.7 \%)$ & $0.62(0.23-1.55)$ & 0.305 \\
\hline \multicolumn{2}{|l|}{$>500 \mathrm{~kb}$ deletion } & \multicolumn{2}{|c|}{$12(2.8 \%)$} & $10(2.4 \%)$ & $1.14(0.50-2.69)$ & 0.750 \\
\hline \multicolumn{2}{|l|}{$>1 \mathrm{Mb}$ deletion } & \multicolumn{2}{|c|}{$5(1.2 \%)$} & $1(0.2 \%)$ & $3.55(0.70-34.68)$ & 0.131 \\
\hline \multicolumn{2}{|l|}{$>500 \mathrm{~kb}$ duplication } & \multicolumn{2}{|c|}{$34(7.9 \%)$} & $31(7.5 \%)$ & $1.05(0.64-1.75)$ & 0.838 \\
\hline \multicolumn{2}{|l|}{$>1 \mathrm{Mb}$ duplication } & \multicolumn{2}{|c|}{$8(1.9 \%)$} & $14(3.4 \%)$ & $0.55(0.22-1.28)$ & 0.168 \\
\hline
\end{tabular}

Table 2: Association of genetic liability for schizophrenia with TRP. Columns for schizophrenia PRS represent the $p$-value threshold used in discovery cohort to derive scores, odds ratio (OR) and $95 \%$ confidence intervals, $R^{2}$ calculated on the liability scale ${ }^{25}$, area under the curve (AUC), standard error (SE), and P-value of association of each score of with TRP. Columns for CNV analysis represent CNVs assessed, frequencies of each CNV in non-TRP (reference group), TRP, odds ratio (OR), 95\% confidence intervals $(\mathrm{Cl})$ and P-value from Firth's logistic regression. 
Figure 1: Variable importance from conditional inference forests model

A

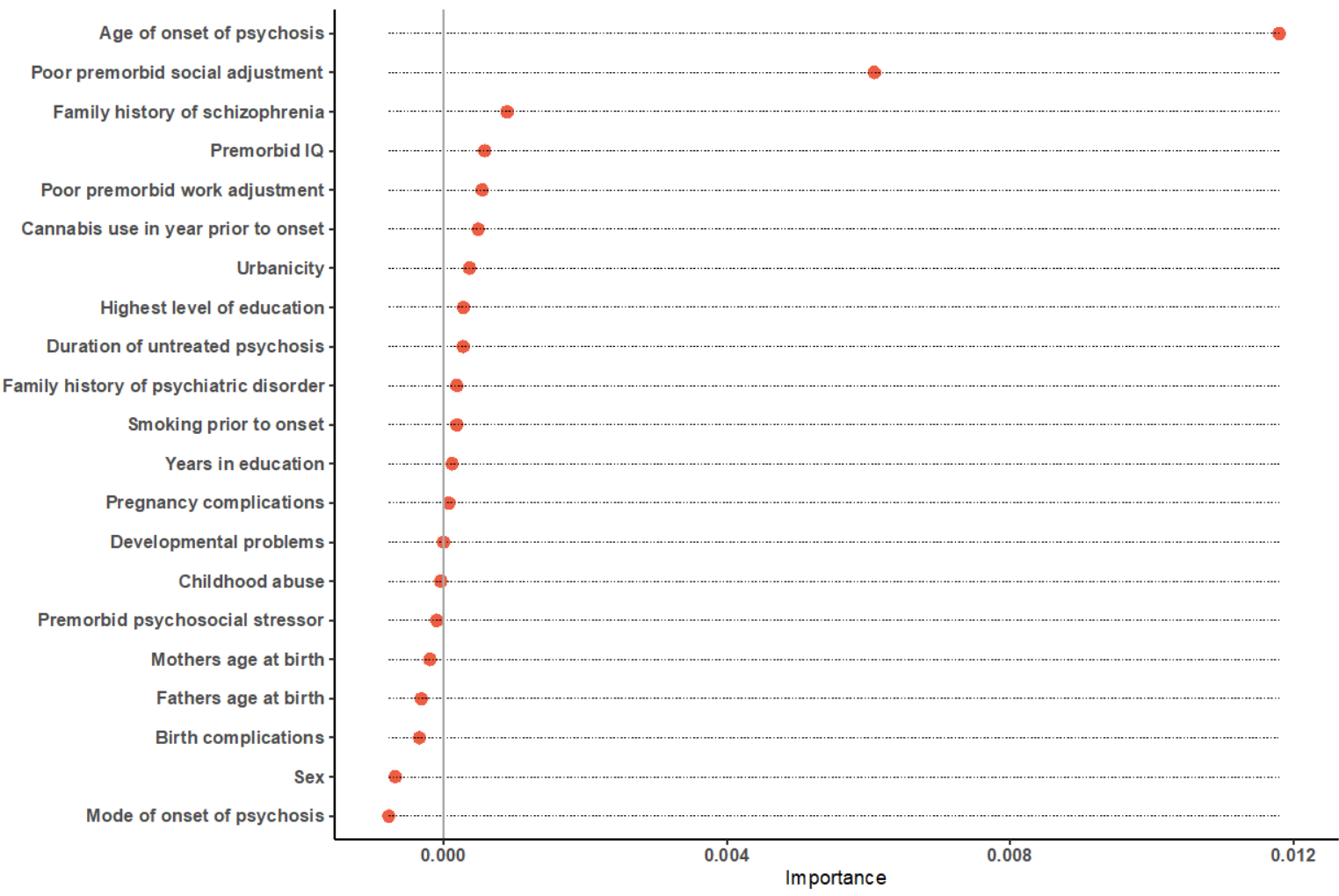

B

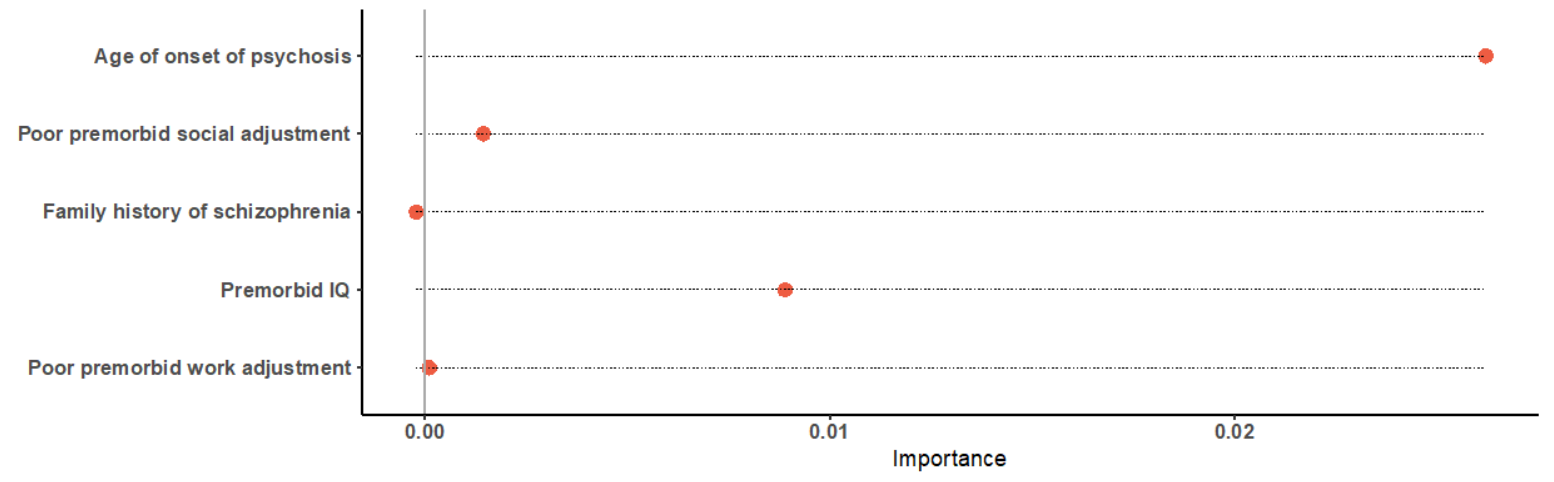

Figure 1: Variable importance plots from conditional inference forests models predicting TRP. Plot A: Permuted importance from primary analysis ( $n=337$ with complete data for all 21 variables assessed). Plot B: Permuted importance from replication analysis for top five variables in remaining sample $(n=428)$. 
Figure 2: Proportions of individuals with TRP by age of onset of psychosis

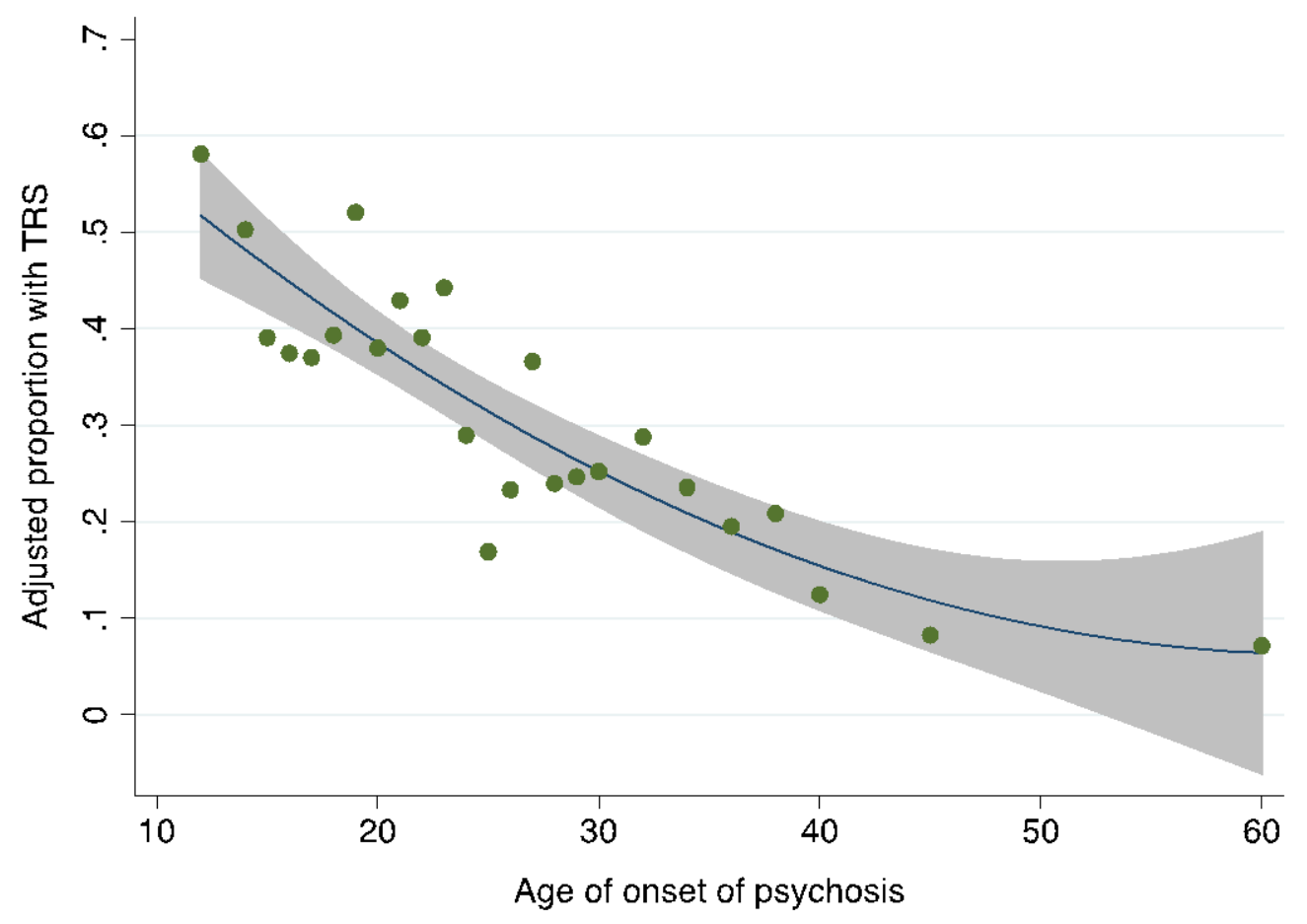

Figure 2: Quadratic fit plot of proportion of sample that have treatment-resistant schizophrenia (TRP). Green dots represent proportion of sample with TRP for each age of onset. Grey area represents $95 \%$ confidence intervals. Data used to produce plot is provided in Supplementary Table 11; the minimum number of per group was 23 individuals, and the mean average number per group was 40 individuals. 